\title{
Kalite Test Fonksiyonları Kullanılarak Güncel Metasezgisel Optimizasyon Algoritmalarının Karşılaştırılması
}

\author{
Soner Kızıloluk ${ }^{1 *}$, Ümit Can ${ }^{2}$ \\ ${ }^{1}$ Malatya Turgut Özal Üniversitesi, Mühendislik ve Doğa Bilimleri Fakültesi, Bilgisayar Mühendisliği Bölümü, \\ Malatya, Türkiye \\ ${ }^{2}$ Munzur Üniversitesi, Mühendislik Fakültesi, Bilgisayar Mühendisliği Bölümü, Tunceli, Türkiye \\ *soner.kiziloluk@ozal.edu.tr ${ }^{\text {(D) }}$,ucan@munzur.edu.tr ${ }^{\text {D }}$ \\ Makale gönderme tarihi: 07.01.2021, Makale kabul tarihi: 26.02.2021
}

\begin{abstract}
$\ddot{O} \mathbf{z}$
Doğadaki canlıların sürü davranışlarından, bitkilerden, insana özgü olgulardan, fizik, matematik, biyoloji ve kimya gibi bilimsel alanlardaki olaylardan esinlenen onlarca metasezgisel optimizasyon yöntemi mevcuttur. Bu yöntemler belirli problemlerde başarılı olmakla birlikte bütün problemlerde başarılı olamamaktadır. Bundan dolayı araştırmacılar tarafından her geçen gün yeni metasezgisel yöntemler önerilmektedir. Bu çalışmada ilk defa güncel Yapay Deniz Anası Optimizasyonu, Etçil Bitki Optimizasyonu, Giza Piramitleri İnşaatı Optimizasyonu, Gradyan Tabanlı Optimizasyon, Öğrenci Psikolojisine Dayalı Optimizasyon ve Tunik Sürüsü Optimizasyonu olmak üzere altı güncel metasezgisel optimizasyon algoritması 10 adet matematiksel kalite test foksiyonunda 10, 30 ve 50 boyut değerleri baz alınarak ayrıntılı bir şekilde karşılaştırılmıştır. Elde edilen sonuçlara göre 10 kalite testinden 7'sinde en iyi sonuçları Öğrenci Psikolojisine Dayalı Optimizasyon vermiştir. Gradyan Tabanlı Optimizasyon'un ise 4 kalite testinde en iyi sonuçları verdiği görülmüştür. En kötü performansı ise Etçil Bitki Optimizasyonu ve Tunik Sürüsü Optimizasyonu göstermiştir. Süre bakımından karşılaştırmak üzere algoritmalar 50 boyutlu test fonksiyonlarında 1000 iterasyonda çalıştırılmış ve elde edilen ortalama çalışma süreleri incelendiğinde, Yapay Deniz Anası Optimizasyonu ve Tunik Sürüsü Optimizasyonu'nun en hızlı çalışan algoritmalar olduğu görülmektedir. Etçil Bitki Optimizasyonu ve Öğrenci Psikolojisine Dayalı Optimizasyon ise en yavaş çalışan algoritmalar olmuştur.
\end{abstract}

Anahtar kelimeler: Global optimizasyon, kalite test fonksiyonları, metasezgisel algoritmalar

\section{Comparison of Current Metaheuristic Optimization Algorithms by Using Benchmark Functions}

\begin{abstract}
There are dozens of metaheuristic optimization methods inspired by the swarm behaviors of creatures in nature, plants, human-specific phenomena, events in scientific fields such as physics, mathematics, biology and chemistry. These methods are successful in certain problems but not in all problems. Therefore, new metaheuristic methods are suggested by researchers frequently. In this study, for the first time, six up-to-date metaheuristic optimization algorithms, namely Jellyfish Search Optimizer, Carnivorous Plant Algorithm, Giza Pyramids Construction Algorithm, Gradient Based Optimizer, Student Based Psychology Optimization and Tunicate Swarm Optimization, were compared by using 10 mathematical benchmark functions with 10, 30 and 50 dimensions. According to the results obtained, Student Based Psychology Optimization gave the best results in 7 out of 10 benchmark functions. It was observed that Gradient Based Optimizer gave the best results in 4 benchmark functions. Carnivorous Plant Algorithm and Tunicate Swarm Optimization showed the worst performance. In order to compare in terms of time, algorithms were run at 1000 iterations in 50dimensional benchmark functions. When the average run times obtained are examined, it is seen that Jellyfish Search Optimizer and Tunicate Swarm Optimization are the fastest running algorithms. Carnivorous Plant Algorithm and Student Based Psychology Optimization were the slowest running algorithms.
\end{abstract}

Keywords: Benchmark functions, global optimization, metaheuristic algorithms 


\section{GİRIS}

Çeșitli bilim ve mühendislik alanlarındaki birçok gerçek dünya uygulaması optimizasyon problemlerine dönüştürülebilir. Çözülmeye çalışılan bu problemler doğrusal olmayan, çok modlu ve oldukça karmaşık problemlerdir. Bir problemin çözüm uzayının tümünün değerlendirilemeyeceği ve sonsuz büyüklükte olduğu durumlarda optimum çözüme en yakın sonucun makul bir zaman diliminde bulunabilmesi için metasezgisel optimizasyon algoritmaları kullanılmaktadır. Metasezgisel optimizasyon algoritmaları oldukça popüler yöntemlerdir ve bunun dört önemli nedeni vardır; (a) Basit konseptlere dayanmaktadırlar ve uygulanmaları kolaydır; (b) amaç fonksiyonu sınırlayıcıların ve kullanılan değişkenlerin tipine bağlı değildir; (c) yerel minimum noktasını baypas edebilirler; ve (d) çeșitli alanlardaki değișik problemleri çözmek için kullanılabilirler (Altunbey ve Alataş, 2015; Mirjalili ve Lewis, 2016; Gao ve Silva, 2018; Chou ve Truong, 2021). Metasezgisel optimizasyon algoritmaları esinlendikleri alanlara göre evrimsel tabanlı, fizik tabanl1, kimya tabanlı, sürü tabanlı, insana dayalı ve bitki tabanlı olmak üzere temel olarak altı sınıfa ayrilabilir.

Evrimsel yöntemler içindeki en popüler olanları Genetik Algoritma (Holland, 1992) ve Diferansiyel Gelişim Algoritmasıdır (Storn ve Price, 1997). Fizik kurallarından veya kanunlarından esinlenilerek geliştirilen fizik tabanlı metasezgisel optimizasyon algoritmalarına Elektromanyetizma Benzeri Algoritma (Birbil ve Fang, 2003), Yerçekimsel Arama Algoritması (Rashedi ve ark., 2009), Parçacık Çarpışma Algoritması (Sacco ve ark., 2005) ve Yapay Fizik Algoritması (Xie ve ark., 2009) örnek olarak verilebilir. Kimya tabanlı yöntemlere ise Yapay Kimyasal Reaksiyon Optimizasyon (Alatas, 2012) algoritması örnek verilebilir. Doğada hayvanların sosyal davranışlarını taklit ederek ve onların sürü zekâsını kullanarak geliștirilen yöntemler sürü tabanlı algoritmalar olarak adlandırılırlar. Bunlar güve, arı, kuş, kedi, kurt ve balina gibi hayvanların davranışlarından esinlenen algoritmalardır. Parçacık Sürü Optimizasyonu (Kennedy ve Eberhart, 1995), Yapay Arı Kolonisi (Karaboga ve Akay, 2009) ve Yarasa Algoritmas1 (Yang ve Gandomi, 2012) gibi algoritmalar sürü tabanlı algoritmalara örnek olarak verilebilir. Spor, müzik, eğitim-öğretim ve yönetim biçimi gibi çeşitli insana dayalı kaynaklardan esinlenerek geliştirilen birçok algoritma bulunmaktadır. Lig Şampiyonası Algoritması (Kashan, 2014) ve Altın Top Optimizasyonu (Osaba ve ark., 2014) spordan esinlenen algoritmalara örnek olarak verilebilir. Harmoni Arama (Lee ve Geem., 2005) ve Melodi Arama (Ashrafi ve Dariane, 2011) algoritmaları müzikten esinlenen yöntemlerdir. Parlamenter sistemden esinlenen Parlamenter Optimizasyon Algoritmas1 (Borji ve Hamidi, 2009) parlamenter sistemin işleyişini simule etmiştir. Bir öğretmenin sınıftaki öğrencilerin öğrenme üzerindeki etkisinden esinlenilerek Öğretme-öğrenmeye dayalı optimizasyon algoritmas1 (Rao ve ark., 2012) geliştirilmiştir. Tüm bu kategorilerin dışında araştırmacılar tarafından bitki davranışlarından esinlenen bitki tabanlı algoritmalar karmaşık problemlerin çözümü için önerilmiştir. Kök kütlesi optimizasyon algoritmas1 (Qi ve ark., 2013; Can ve Alataş, 2015) ve Çiçek Tozlaşması Algoritması (Yang, 2012) bu tür tekniklere örnektir.

Araştırmacılar tarafindan yeni birçok optimizasyon algoritması geliştirilmesine rağmen hala bu zorlu problemlerin çözümünde tatmin edici sonuçlar ortaya koymada yeterli olamamaktadırlar (Ahmadianfar ve ark., 2020). Optimizasyon problemlerine daha başarılı çözümler sunmak için araştırmacılar tarafindan yeni sezgisel yöntemler önerilmektedir. Yapay Deniz Anası Optimizasyonu (YDAO) (Chou ve Truong, 2021), Etçil Bitki Optimizasyonu (EBO) (Ong ve ark., 2021), Giza Piramitleri İnşaatı Optimizasyonu (GPİO) (Harifi ve ark., 2020), Gradyan Tabanlı Optimizasyon (GTO) (Ahmadianfar ve ark., 2020), Öğrenci Psikolojisine Dayalı Optimizasyon (ÖPDO) (Das ve ark., 2020) ve Tunik Sürüsü Optimizasyonu (TSO) (Kaur ve ark., 2020) 2020 y1lı içerisinde önerilmiş güncel metasezgisel yöntemlerdir. Bu çalışmada son bir yıl içerisinde önerilen güncel altı adet metasezgisel algoritma incelenmiş ve bu algoritmalar ilk defa on adet sıkça kullanılan kalite test fonksiyonu kullanılarak karşılaştırılmıştır. Elde edilen sonuçlar detaylı bir şekilde verilmiştir.

Giriş bölümünde metasezgisel optimizasyon yöntemleri anlatılarak literatürdeki mevcut çalışmalar hakkında bilgi verilmiștir. Güncel Metasezgisel Optimizasyon Algoritmaları başlığında ise çalışmada kullanılan güncel metasezgisel algoritmaların esinlenme kaynakları verilerek bu algoritmaların kaba kodları gösterilmiştir. Deneysel Çalışma ve Sonuçlar bölümünde algoritmalar on adet matematiksel kalite test fonksiyonu kullanılarak 
karşılaştırılmış ve sonuçlar ayrıntılı bir şekilde verilmiştir. Sonuç bölümünde ise deneysel çalışmalar sonucunda elde edilen sonuçlar değerlendirilmiştir

\section{GÜNCEL METASEZGISEL OPTIMIZASYON ALGORITMALARI}

Bilimsel araştırmalar sürdükçe insanların keşifleri artmakta bu da yeni metasezgisel algoritmalar geliştirilmesi için farklı esin kaynaklarının ortaya çıkmasını sağlamaktadır. Bu bölümde, deneysel çalışmada karşılaştırmaları yapılan 6 farklı güncel metasezgisel optimizasyon algoritmasının temel esin kaynakları ve algoritma adımlarını içeren kaba kodları alt başlıklarda verilmiştir.

\section{Yapay Denizanası Arama Optimizasyonu}

Denizanaları çeşitli boyutlarda ve çeşitli renklerde var olan ve farklı sicaklıklardaki sularda farklı derinliklerde yaşayabilen canlılardır. Denizanalarının beslenme yöntemleri birbirinden farklıdır. Bazı denizanaları yiyecekleri ağızlarına götürmek için dokunaçlarını kullanırken, bazıları ise akıntıların getirdikleri ile beslenmek için filtreleme yöntemini kullanırlar. Bazı denizanaları avlarını aktif olarak avlar ve dokunaçlarıyla sokarak onları hareketsiz hale getirirler. Yapay denizanası arama optimizasyon (YDAO) algoritmas1 denizanalarının okyanuslardaki yiyecek arama davranışından esinlenilmiş ve matematiksel olarak bir denizanası topluluğu modellenmiştir. $\mathrm{Bu}$ modeli temel alan algoritma üç kural üzerine kurulmuştur (Chou ve Truong, 2021).

a) Bir denizanası ya okyanus akıntısını takip eder ya da sürü içinde hareket eder ve bir "zaman kontrol mekanizması" bu hareketler arasındaki geçişi belirler.

b) Denizanası yiyecek aramak için okyanusta hareket eder. Mevcut yiyecek miktarının daha fazla olduğu yerlere daha çok çekilir.

c) Bulunan yiyeceğin miktarına konum ve konumun uygunluk fonksiyonu karar verir.

YDAO algoritmasının temel adımları Algoritma 1' deki kaba kodda verilmiştir.

\section{ALGORITMA 1 \\ START}

Arama uzay1 sınırlarını, popülasyon sayıs1 $\left(N_{p o p}\right)$ ve maksimum iterasyon (Max it) sayısını ayarla. Başlangıç popülasyonunu oluştur.
Popülasyondaki herbir denizanasının uygunluk değerini hesapla.

Uygunluğu en iyi (En çok yiyeceğin bulunduğu) denizanasını $X^{*}$ olarak tanımla.

İterasyonu $t=1$ olarak ayarla.

\section{DO}

FOR1 $i=1: N_{p o p}$

Zaman kontrolü $c(t)^{\prime}$ yi hesapla.

IF1 $c(t) \geq 0.5$ : (Denizanas1 okyanus akıntısını takip eder)

(1) Okyanus akıntısına karar ver.

(2) Denizanasının yeni konumuna karar ver.

ELSE (Denizanası sürü içinde hareket eder)

IF2 $\operatorname{rand}(0,1)>(1-c(t))$

(1) Denizanasının yeni konumuna karar ver.

ELSE

(2) Denizanasının yönüne karar ver.

(3) Denizanasının yeni konumuna karar ver.

END IF2

\section{END IF1}

Sınır şartlarını kontrol et ve yeni noktadaki yiyecek miktarını bul

Mevcut denizanasının konumunu ve en çok yiyeceğe sahip denizanasının $\left(X^{*}\right)$ konumunu güncelle.

\section{END FOR1}

$t=t+1$.

WHILE $t>$ Max it

En iyi deniz anasını problemin çözümü olarak kabul et.

\section{STOP}

\section{Etçil Bitki Optimizasyonu}

Bitkilerin birçoğu hayvanlar için besin kaynağ 1 konumunda iken etçil bitkiler için bu durum tam tersidir. Etçil bitkiler zor şartlarda hayatta kalmanın yanı sıra sinek, kelebek, kertenkele ve fare gibi hayvanları avlar (Ong ve ark., 2021). Bu noktada Etçil Bitki Optimizasyon (EBO) algoritması etçil bitkilerin zorlu şartlarda hayatta kalmak için avlanma davranışlarından ve üremek için tozlaşma adaptasyonlarından esinlenilerek önerilmiştir. Optimizasyon için etçil bitkilerin çekicilik, tuzağa düşürme, sindirme ve üreme stratejileri matematiksel olarak modellenmiştir. Algoritma 2' de EBO algoritmasının kaba kodu gösterilmektedir. 


\section{ALGORITMA 2 \\ START}

grup_iter, cekim_orani, buyume_orani, ureme_orani, $n E B i t k i$ ve $n A v$ değişkenlerini tanımla.

$d$ boyutlu ve $n$ bireyli rastgele başlangıç popülasyonu oluştur.

Uygunluk değerlerine göre bireyleri sirala.

En iyi bireyi $g *$ olarak tanımla.

WHILE (Bitim şartı sağlanıncaya kadar)

En iyi bireyleri etçil bitkiler olarak sınıflandır (nEBitki).

Geriye kalan bireyleri av olarak sinıflandır $(n A v)$.

Etçil bitkileri ve avları gruplandır

FOR1 $i=0: n E B i t k i$

FOR2 $j=1$ : grup_iter

IF1 cekim_orani $>$ rastgele üretilen sayı

Yeni bir etçil bitki üret.

\section{ELSE}

Yeni bir av üret.

\section{END IF1}

END FOR2

END FOR1

FOR3 $i=1: n E B i t k i$

Yeni bir etçil bitki üret.

\section{END FOR3}

Her bir yeni etçil bitkinin ve yeni avların uygunluğunu hesapla.

Eski ve yeni üretilmiş etçil bitkileri ve avları birleștir.

Bireyleri uygunluk değerlerine göre sırala ve en iyi $\mathrm{n}$ adet bireyi sonraki nesle aktar. $g^{*}$ ' 1 güncelle.

\section{END WHILE}

En iyi bireyi $\left(g^{*}\right)$ problemin çözümü olarak kabul et.

\section{STOP}

\section{Giza Piramitleri İnşaatı Optimizasyonu}

Giza Piramitleri eski Mısır'da inșa edilmiș üç büyük piramitten oluşan bir yapı bloğudur. Arkeologlara göre birbirinden farklı büyüklükteki bu piramitlerin yapımı zamana yayıldığı için yapım yöntemleri birbirinden farklıdır. $\mathrm{Bu}$ inşaatların yapımında en önemli konulardan biri her biri inşaat alanında farklı sorumluluklara sahip hamallar, köleler, masonlar, metal işçileri ve marangozların nasıl yönetileceği meselesi idi. Bunlar firavunun ajanı tarafından yönlendirilirlerdi. Ayrıca kullanılan inşaat malzemelerinin kısıtlılığ 1 , inşaat süresi sorunu ve kullanılan taş bloklar nedeniyle piramitlerin yapımı optimize edilmiştir. Giza Piramitleri İnşaatı Optimizasyon (GPIO) algoritmas1 bu piramitlerin yapıldığ1 dönemdeki metotları, teknolojileri ve stratejileri gözlemleyip bunlardan esinlenen bir algoritma olarak ortaya çıkmıştır (Harifi ve ark., 2020). Algoritma 3' te GPİO algoritmasının kaba kodu verilmiştir.

\section{ALGORITMA 3 \\ START}

Rastgele üretilmiş $n$ adet taş bloklar veya işçiler ile başlangıç popülasyonunu oluştur.

Taş blok ve işçilerin uygunluk değerini hesapla.

En iyi işçiyi firavunun ajanı olarak belirle.

FOR1 $I t=1:$ MaxIt

FOR2 $i=1: n$

Taş blok yer değiştirme miktarını hesapla. İşçi hareketinin miktarını hesapla.

Yeni pozisyonu tahmin et.

İşçileri ikame etme olasılığını araştır.

Yeni pozisyon ve uygunluk değerlerini hesapla.

IF1 yeni uygunluk değeri< Firavunun ajanı uygunluk değeri

Yeni uygunluk değerini firavunun ajanı uygunluk değeri olarak ayarla.

\section{END IF1}

END FOR2

\section{END FOR1}

\section{STOP}

\section{Gradyan Tabanlı Optimizasyon}

Gradyan Tabanlı Optimizasyon (GTO) algoritmas1 Newton'un gradyan tabanlı metodundan esinlenilerek önerilmiştir (Ahmadianfar ve ark., 2020). Newton'un metodu denklemleri sayısal olarak çözen güçlü bir metottur ve bu yöntem, Taylor serisinin ilk terimlerini kullanan bir kök bulma algoritmasıdır. $\mathrm{Bu}$ metottan esinlenen GTO algoritmasının işleyişi sırasında iki önemli operatör kullanılır. Bunlar gradyan arama kuralı (GAK) ve yerel kaçış operatörü (YKO) olarak adlandırılırlar. GAK, arama uzayında keşif eğilimini arttırmak ve yakınsama oranını hızlandırmak için gradyan tabanlı yöntemi kullanır. YKO ise GTO algoritmasının yerel minimumdan kaçınması için kullandığı bir operatör işlevi görmektedir (Ahmadianfar ve ark., 2020). 
Algoritma 4' te GTO algoritmasının kaba kodu gösterilmektedir.

\section{ALGORITMA 4 \\ START}

$p r, \varepsilon$ ve $M$ parametrelerinin değerlerini ata.

Başlangıç popülasyonunu oluştur.

Popülasyondaki her elemanın uygunluk değerini hesapla.

En iyi ve en kötü elemanı belirle.

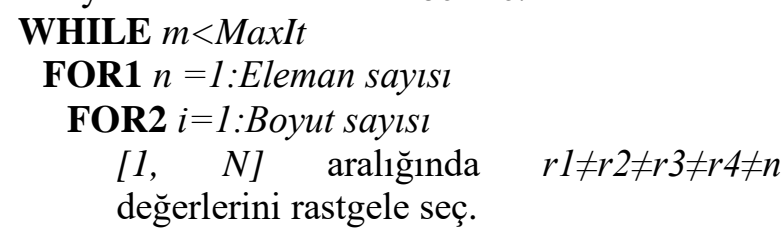

Elemanın yeni pozisyonunu hesapla $\left(X_{n, i}^{m}\right)$.

\section{END FOR2}

//Yerel kaçış operatörü (YKO)

IF random $<p r$

$X_{Y K O}^{m}$ nun pozisyonunu hesapla. $X^{m+1}{ }_{n, i}=X^{m}{ }_{\text {YKO. }}$

END IF

En iyi ve en kötü elemanı güncelle.

\section{END FOR1}

$m=m+1$.

\section{END WHILE}

STOP

\section{Öğrenci Psikolojisine Dayalı Optimizasyon}

Öğrenci Psikolojisine Dayalı Optimizasyon (ÖPDO) algoritması Hindistan'ın Batı Bengal bölgesindeki öğrencilerin psikolojileri üzerine yapılan çalışmalar sonucu elde edilen veriler üzerine ortaya çıkmıştır (Das ve ark., 2020). ÖPDO algoritması, bir sınıfın en iyisi olmaya çabalayan bir öğrencinin psikolojisine dayanarak önerilmiştir. Öğrenciler bir sınavda başarılı notlar almak ister fakat bu öğrencilerin verimliliğine ve derse olan ilgilerine bağlıdır. Bu psikolojiye bağlı olarak öğrenciler bir sınıf içerisinde en başarılı öğrenci olmak için çabalarlar (Das ve ark., 2020). Algoritma 5' te ÖPDO'nun kaba kodu verilmiştir.

\section{ALGORTIMA 5 \\ START}

Popülasyonu ve yakınsama kriterini oluştur.

Sınıfin başlangıç performansını hesapla.

WHILE $P<=$ MaxIt

FOR1 $m=1$ :önerilen konu saylsı

// Öğrencilerin katagorilerinin kontrolü

\section{IF1 ögrenci=en iyi}

En iyi öğrencinin performansını güncelle.

ELSE IF2 ögrenci=iyi

//her öğrenci için, öğrencinin yalnızca en iyi öğrenciyi takip edip etmediğinin kontrolü.

IF3 Evet

Mevcut öğrencinin performansını en iyi öğrencinin performansı doğrultusunda güncelleştir.

\section{ELSE}

Mevcut öğrencinin performansını en iyi öğrencinin performans1 ve tüm öğrencilerin performanslarının ortalaması doğrultusunda güncelleștir.

END IF3

END ELSE IF2

ELSE IF4 ögrenci $=$ ortalama

Mevcut öğrencinin performansını tüm öğrencilerin performanslarının ortalaması doğrultusunda güncelleştir.

\section{END ELSE IF4}

ELSE

Mevcut öğrencinin performansını gelişigüzel değerler doğrultusunda güncelleştir.

\section{END IF1}

Sinırları kontrol et.

Sinıfin yeni performansını hesapla.

IF5 yeni performans > Eski performans

Eski performansı yeni performans ile değiştir

ELSE

Eski performansı koru

\section{END IF5}

$m=m+1$

\section{END FOR1}

\section{$P=P+1$}

\section{END WHILE}

\section{STOP}

\section{Tunik Sürüsü Optimizasyonu}

Tunik Sürüsü Optimizasyonu (TSO) bir deniz canlısı olan tuniklerin yön bulma ve beslenme davranışlarından esinlenen sürü tabanlı bir algoritmadır (Kaur ve ark., 2020). Tuniklerin denizdeki besin kaynaklarının yerini bulma yetenekleri vardır. Burada besin kaynaklarını bulmak için tunikler jet itme hareketi ve sürü zekâsı olmak üzere iki davranış sergiler. Optimizasyon için jet itme hareketi matematiksel olarak modellenmiştir. Bunun için arama ajanlarının birbirleri ile çatışmalarını engellemek, en iyi arama ajanına doğru hareket etmek 
ve en iyi ajana yakın kalmak gibi üç şart sağlanmalıdır. Sürü davranışı da diğer bireylerin en iyi çözüme göre kendi konumlarını güncellemeleri ile gerçekleşir (Kaur ve ark., 2020). Algoritma 6' da TSO algoritmasının kaba kodu verilmiştir.

\section{ALGORITMA 6}

\section{START}

Başlangış popülasyonunu rastgele tunikler ile oluştur.

Başlangıç parametre değerlerini belirle.

Her bir tunikin uygunluk değerini hesapla.

Uygunluk değeri en iyi olan tuniki $B E S T_{t}$ olarak belirle.

WHILE (Bitim şartı sağlanıncaya kadar)

Tuniklerin jet itme değerini ve sürü davranışlarını hesapla.

Her tunikin konumunu güncelle.

Tuniklerin arama uzayı sınırlarının dışına çıkıp çıkmadığını kontrol et.

IF1 tunik arama uzayı sınırları dışındamı

Tunikin değerlerini sınırlar içinde kalacak şekilde güncelle.

\section{END IF1}

Konumu güncellenen tuniklerin uygunluk değerlerini hesapla.

Eğer bir önceki iterasyondaki en iyi uygunluk değerine sahip tunikten $\left(B E S T_{t}\right)$ daha iyi uygunluk değerine sahip bir tunik mevcut ise, o tuniki popülasyonun en iyi tuniki $\left(B E S T_{t}\right)$ olarak güncelle.

\section{END WHILE}

Uygunluk değeri en iyi tuniki problemin çözümü olarak kabul et.

\section{STOP}

\section{DENEYSEL ÇALIŞMA VE SONUÇLAR}

$\mathrm{Bu}$ bölümde güncel metasezgisel optimizasyon algoritmalarının karşılaştırılması için kullanılan kalite test fonksiyonları anlatılmış ve bu fonksiyonlar kullanılarak elde edilmiş sonuçlar ayrıntılı bir şekilde verilmiştir.

\section{Kullanılan Kalite Test Fonksiyonları}

Sezgisel optimizasyon algoritmalarının performanslarını değerlendirebilmek amacıyla literatürde bir çok matematiksel kalite test fonksiyonu mevcuttur. $\mathrm{Bu}$ fonksiyonlar gerçek hayattaki mühendislik problemlerinin zorluk ve karmaşıklığına sahiptirler. $\mathrm{Bu}$ fonksiyonlar optimizasyon algoritmalarını yakınsama, hassasiyet, sağlamlık ve genel performans açısından değerlendirmek ve karşılaştırmak için sıklıkla kullanılır. Bu kıyaslama fonksiyonlarının doğal işlevleri, karmaşıklıkları ve diğer özellikleri tanımlarından elde edilebilir ve bu fonksiyonlarının zorluk seviyeleri, boyut ve aralık parametreleri değiştirilerek ayarlanabilir (Alatas ve ark., 2009; Kızıloluk ve Özer, 2016).

Tablo 1. Kullanilan kalite test fonksiyonlart

\begin{tabular}{|l|l|l|l|l|}
\hline Fonksiyon & Denklem & Tür & Sinır & Optimum \\
\hline Sphere (F1) & $\sum_{i=1}^{d} x_{i}^{2}$ & TM & \pm 100 & 0 \\
\hline Rosenbrock (F2) & $\sum_{i=1}^{d-1}\left[100\left(x_{i}^{2}-x_{i+1}\right)^{2}+\left(1-x_{i}^{2}\right)\right]$ & TM & \pm 30 & 0 \\
\hline Step (F3) & $\sum_{i=1}^{d}\left(x_{i}+0.5\right)^{2}$ & TM & \pm 100 & 0 \\
\hline Rastrigin (F4) & $10 d+\sum_{i=1}^{d}\left[x_{i}^{2}-10 \cos \left(2 \pi x_{i}\right)\right]$ & ÇM & \pm 5.12 & 0 \\
\hline Ackley (F5) & $\begin{array}{l}20+e-20 \exp \left(-0.2 \sqrt{\frac{1}{d} \sum_{i=1}^{d} x_{i}^{2}}\right)- \\
\left.\text { exp }\left(\frac{1}{d} \sum_{i=1}^{d} \cos \left(2 \pi x_{i}\right)\right)\right)\end{array}$ & ÇM & \pm 32 & 0 \\
\hline Griewank (F6) & $\sum_{i=1}^{d}\left(\frac{x_{i}^{2}}{4000}\right)-\prod_{i=1}^{d} \cos \left(\frac{x_{i}}{\sqrt{i}}\right)+1$ & ÇM & \pm 600 & 0 \\
\hline Levy (F7) & $\begin{array}{l}\sin ^{2}\left(\pi y_{1}\right)+\sum_{i=1}^{d-1}\left(y_{i}-1\right)^{2}\left[1+10 \sin ^{2}\left(\pi y_{i+1}\right)\right]+ \\
\left(y_{n}-1\right)^{2}, y_{i}=1+\frac{x_{i}-1}{4}\end{array}$ & ÇM & \pm 10 & 0 \\
\hline Alpine (F8) & $\sum_{i=1}^{d}\left|x_{i} \sin \left(x_{i}\right)+0.1 x_{i}\right|$ & ÇM & \pm 10 & 0 \\
\hline Quintic (F9) & $\sum_{i=1}^{d}\left|x_{i}^{5}-3 x_{i}^{4}+4 x_{i}^{3}+2 x_{i}^{2}-10 x_{i}-4\right|$ & ÇM & \pm 10 & 0 \\
\hline Trigonometric (F10) & $\begin{array}{l}\sum_{i=1}^{d} 8 \sin ^{2}\left[7\left(x_{i}-0.9\right)^{2}\right]+6 \sin ^{2}\left[14\left(x_{i}-0.9\right)^{2}\right]+ \\
\left(x_{i}-0.9\right)^{2}\end{array}$ & ÇM & \pm 500 & 0 \\
\hline
\end{tabular}


$\mathrm{Bu}$ çalışmada 10 adet matematiksel test fonksiyonu kullanılmıştır. Kullanılan kalite fonksiyonlarının denklem, tür, sınır değerleri ve optimum değer bilgileri Tablo 1' de verilmiştir (Jamil ve Yang, 2013). Tablodaki denklemlerde $x$ değerleri değişkenleri $d$ değeri ise problem boyutunu temsil etmektedir. Bu fonksiyonlardan sphere, rosenbrock ve step Tek Modlu (TM) yani sadece tek bir global optimuma sahip ve geri kalan fonksiyonlar ise Çok Modlu (ÇM) yani tek bir global optimuma, birden çok yerel optimuma sahip.

\section{Deneysel Sonuçlar}

Tüm deneysel çalışmalar MATLAB 2020b platformu üzerinde gerçekleştirilmiştir. Çalışmada karşılaştırılan tüm sezgisel algoritmalar için başlangıç popülasyon sayıları 30 ve maksimum iterasyon sayısı 1000 olarak alınmıştır. Fonksiyonların boyut değerleri 10,30 ve 50 alınarak algoritmalar 3 farklı zorlukta test edilmiştir. Her test fonksiyonu için algoritmaların performansları, 30 bağımsız çalışmada elde edilen sonuçlara göre değerlendirilmiştir. Elde edilen karşılaştırmalı test sonuçları 10 boyutlu fonksiyonlar için Tablo 2' de, 30 boyutlu fonksiyonlar için Tablo 3' te ve 50 boyutlu fonksiyonlar için Tablo 4' te verilmiştir. Tablolarda 30 bağımsız çalıştırmada elde edilen ortalama, en iyi ve standart sapma değerleri verilmiştir. Ortalama ve en iyi değerler algoritmaların global optimuma yakınsama performansını temsil eder. Standart sapma ise algoritmanın kararlı çalışıp çalışmadığını gösterir.

Tablo 2. 10 boyutlu test fonksiyonlarında elde edilen sonuçlar

\begin{tabular}{|c|c|c|c|c|c|c|c|}
\hline & & YDAO & EBO & GPIO & GTO & ÖPDO & TSO \\
\hline \multirow[t]{3}{*}{ F1 } & Ort. & $2.63 \mathrm{E}-84$ & $3.50 \mathrm{E}-76$ & $8.02 \mathrm{E}-25$ & $6.09 \mathrm{E}-261$ & $1.45 E-289$ & $7.31 \mathrm{E}-84$ \\
\hline & En iyi & $4.43 \mathrm{E}-94$ & $6.80 \mathrm{E}-84$ & $1.31 \mathrm{E}-29$ & $9.87 \mathrm{E}-281$ & $2.60 \mathrm{E}-294$ & $2.45 \mathrm{E}-90$ \\
\hline & Std. & $9.86 \mathrm{E}-84$ & $1.68 \mathrm{E}-75$ & $3.12 \mathrm{E}-24$ & 0 & 0 & $2.01 \mathrm{E}-83$ \\
\hline \multirow[t]{3}{*}{ F2 } & Ort. & 0.06227 & 2.73034 & 7.19726 & 0.00185 & 0.07724 & 13.49050 \\
\hline & En iyi & $6.14 \mathrm{E}-08$ & 0.03693 & 6.54867 & 5.10E-09 & 0.00120 & 5.11381 \\
\hline & Std. & 0.17952 & 3.29056 & 0.26851 & 0.00695 & 0.09705 & 19.49559 \\
\hline \multirow[t]{3}{*}{ F3 } & Ort. & $3.91 \mathrm{E}-27$ & $1.31 \mathrm{E}-32$ & 0.34769 & $2.05 \mathrm{E}-32$ & 0 & 1.16185 \\
\hline & En iyi & $3.76 \mathrm{E}-31$ & 0 & 0.15776 & 0 & 0 & 0.50588 \\
\hline & Std. & $8.33 \mathrm{E}-27$ & $3.80 \mathrm{E}-32$ & 0.10437 & $2.91 \mathrm{E}-32$ & $\mathbf{0}$ & 0.33068 \\
\hline \multirow[t]{3}{*}{ F4 } & Ort. & $2.89 \mathrm{E}-09$ & 10.01591 & 0 & 0 & 0 & 26.04894 \\
\hline & En iyi & 0 & 1.98992 & $\mathbf{0}$ & $\mathbf{0}$ & $\mathbf{0}$ & 10.95320 \\
\hline & Std. & $1.55 \mathrm{E}-08$ & 4.63792 & $\mathbf{0}$ & 0 & 0 & 9.05345 \\
\hline \multirow[t]{3}{*}{ F5 } & Ort. & $1.48 \mathrm{E}-15$ & 0.17039 & $1.64 \mathrm{E}-13$ & 8.88E-16 & $6.57 \mathrm{E}-15$ & 1.05424 \\
\hline & En iyi & $8.88 \mathrm{E}-16$ & $4.44 \mathrm{E}-15$ & $8.88 \mathrm{E}-16$ & $8.88 \mathrm{E}-16$ & $4.44 \mathrm{E}-15$ & $4.44 \mathrm{E}-15$ \\
\hline & Std. & $1.32 \mathrm{E}-15$ & 0.44129 & $2.30 \mathrm{E}-13$ & 0 & $1.74 \mathrm{E}-15$ & 1.57406 \\
\hline \multirow[t]{3}{*}{ F6 } & Ort. & $\mathbf{0}$ & 0.04963 & $\mathbf{0}$ & 0 & 0 & 0.40022 \\
\hline & En iyi & $\mathbf{0}$ & 0 & $\mathbf{0}$ & $\mathbf{0}$ & $\mathbf{0}$ & 0 \\
\hline & Std. & 0 & 0.03303 & 0 & 0 & 0 & 0.25573 \\
\hline \multirow[t]{3}{*}{ F7 } & Ort. & $5.71 \mathrm{E}-27$ & 0.11120 & 0.60254 & $3.16 \mathrm{E}-30$ & 1.50E-32 & 0.83622 \\
\hline & En iyi & $2.10 \mathrm{E}-30$ & $1.50 \mathrm{E}-32$ & 0.45402 & $1.50 \mathrm{E}-32$ & $1.50 \mathrm{E}-32$ & 0.18068 \\
\hline & Std. & $1.95 \mathrm{E}-26$ & 0.34592 & 0.07917 & $8.44 \mathrm{E}-30$ & 0 & 1.49648 \\
\hline \multirow[t]{3}{*}{ F8 } & Ort. & $1.25 \mathrm{E}-08$ & $4.53 \mathrm{E}-16$ & $4.83 \mathrm{E}-15$ & 2.21E-131 & 1.04E-29 & 2.54171 \\
\hline & En iyi & $6.52 \mathrm{E}-45$ & $9.50 \mathrm{E}-111$ & $3.46 \mathrm{E}-21$ & 3.83E-141 & $7.30 \mathrm{E}-71$ & 0.17516 \\
\hline & Std. & $3.30 \mathrm{E}-08$ & $5.88 \mathrm{E}-16$ & $8.36 \mathrm{E}-15$ & 1.18E-130 & $3.12 \mathrm{E}-29$ & 1.61732 \\
\hline \multirow[t]{3}{*}{ F9 } & Ort. & 1.19E-11 & $1.18 \mathrm{E}-15$ & 14.40151 & $1.03 \mathrm{E}-11$ & 0 & 16.24432 \\
\hline & En iyi & 0 & 0 & 10.65436 & 0 & 0 & 3.80510 \\
\hline & Std. & $4.45 \mathrm{E}-11$ & $5.07 \mathrm{E}-15$ & 1.83854 & $5.56 \mathrm{E}-11$ & $\mathbf{0}$ & 5.29426 \\
\hline \multirow[t]{3}{*}{ F10 } & Ort. & $7.29 \mathrm{E}-25$ & 1.15176 & 13.65040 & 4.29291 & 0 & 26.26650 \\
\hline & En iyi & $7.04 \mathrm{E}-30$ & 0 & 4.40142 & 0.44864 & 0 & 2.28676 \\
\hline & Std. & $3.62 \mathrm{E}-24$ & 1.28337 & 3.03316 & 3.13423 & $\mathbf{0}$ & 10.97233 \\
\hline
\end{tabular}


Tablo 2' deki 10 boyutlu test fonksiyonlarından elde edilen sonuçlar incelendiğinde F1, F3, F7, F9 ve F10 fonksiyonlarında en iyi ortalama değerleri ÖPDO' nun verdiği görülmektedir. F2, F5 ve F8' de ise GTO en iyi performansı göstermiştir. F4' de GPIO, GTO ve ÖPDO, F6' da ise YDAO, GPİ, GTO ve ÖPDO en iyi sonuçları vermiştir. En kötü performansı ise TSO ve EBO göstermiştir. 10 boyutlu test fonksiyonlarında toplam 7 fonksiyonda en iyi değerleri veren ÖPDO ilk sırada yer alırken onu 5 fonksiyonda en iyi değerleri vererek GTO takip etmiştir.

Tablo 3' teki 30 boyutlu test fonksiyonlarından elde edilen sonuçlar incelendiğinde toplam 7 fonksiyonda en iyi ortalama ve standart sapma değerlerini veren ÖPDO yine en iyi performans1 göstermiştir. GTO ise toplam 4 fonksiyonda en iyi sonuçları vermiş ve en başarılı ikinci algoritma olmuştur. Bu iki algoritmayı YDAO ve GTO toplam ikișer fonksiyonda en iyi değerleri vererek takip etmiştir. En kötü performansı ise EBO ve TSO göstermiștir.

Tablo 4' teki 50 boyutlu test fonksiyonlarından elde edilen sonuçlar incelendiğinde yine ÖPDO toplam 7 fonksiyonda en iyi değerleri vererek en başarılı algoritma olmuştur. GTO ise toplam 4 fonksiyonda, YDAO ve GPIO ise toplam 2 fonksiyonda en iyi sonuçları vermiştir. 50 boyutlu test fonksiyonlarında da yine en kötü başarıyı EBO ve TSO göstermektedir.

Tablo 3. 30 boyutlu test fonksiyonlarında elde edilen sonuçlar

\begin{tabular}{|c|c|c|c|c|c|c|c|}
\hline & & YDAO & EBO & GPİO & GTO & ÖPDO & TSO \\
\hline \multirow[t]{3}{*}{ F1 } & Ort. & $1.18 \mathrm{E}-46$ & $1.66 \mathrm{E}-15$ & $4.60 \mathrm{E}-22$ & $2.11 \mathrm{E}-241$ & $5.44 \mathrm{E}-273$ & $1.66 \mathrm{E}-47$ \\
\hline & En iyi & $2.40 \mathrm{E}-75$ & $1.35 \mathrm{E}-17$ & $3.74 \mathrm{E}-26$ & $2.68 \mathrm{E}-259$ & 4.10E-276 & $1.16 \mathrm{E}-50$ \\
\hline & Std. & $6.34 \mathrm{E}-46$ & $5.94 \mathrm{E}-15$ & $1.10 \mathrm{E}-21$ & $\mathbf{0}$ & $\mathbf{0}$ & $2.89 \mathrm{E}-47$ \\
\hline \multirow[t]{3}{*}{$\mathrm{F} 2$} & Ort. & 0.10381 & 59.63437 & 27.43257 & 20.81292 & 1.69222 & 28.37646 \\
\hline & En iyi & 4.58E-05 & 4.01466 & 26.85205 & 17.40447 & 0.00169 & 26.17606 \\
\hline & Std. & 0.43619 & 59.80774 & 0.20578 & 1.61170 & 4.74616 & 0.70659 \\
\hline \multirow[t]{3}{*}{ F3 } & Ort. & $9.48 \mathrm{E}-09$ & $1.88 \mathrm{E}-15$ & 4.46893 & 2.92E-09 & 0 & 3.75670 \\
\hline & En iyi & $1.71 \mathrm{E}-12$ & $1.32 \mathrm{E}-17$ & 4.00592 & $4.18 \mathrm{E}-12$ & $\mathbf{0}$ & 2.30009 \\
\hline & Std. & $2.66 \mathrm{E}-08$ & $3.84 \mathrm{E}-15$ & 0.19747 & 7.99E-09 & $\mathbf{0}$ & 0.70124 \\
\hline \multirow[t]{3}{*}{$\mathrm{F} 4$} & Ort. & 0.00392 & 49.91371 & 0 & 0 & $\mathbf{0}$ & 163.36447 \\
\hline & En iyi & $2.27 \mathrm{E}-11$ & 29.84875 & $\mathbf{0}$ & $\mathbf{0}$ & $\mathbf{0}$ & 92.62398 \\
\hline & Std. & 0.01712 & 13.57688 & $\mathbf{0}$ & $\mathbf{0}$ & $\mathbf{0}$ & 36.22485 \\
\hline \multirow[t]{3}{*}{ F5 } & Ort. & $3.49 \mathrm{E}-15$ & 0.86503 & $3.27 \mathrm{E}-12$ & 8.88E-16 & $3.42 \mathrm{E}-14$ & 1.37351 \\
\hline & En iyi & $8.88 \mathrm{E}-16$ & $1.45 \mathrm{E}-09$ & $1.87 \mathrm{E}-14$ & $8.88 \mathrm{E}-16$ & $2.22 \mathrm{E}-14$ & $7.99 \mathrm{E}-15$ \\
\hline & Std. & $1.57 \mathrm{E}-15$ & 1.05580 & $3.58 \mathrm{E}-12$ & $\mathbf{0}$ & $4.72 \mathrm{E}-15$ & 1.48571 \\
\hline \multirow[t]{3}{*}{ F6 } & Ort. & $\mathbf{0}$ & 0.01066 & $\mathbf{0}$ & $\mathbf{0}$ & 0 & 0.01105 \\
\hline & En iyi & $\mathbf{0}$ & $\mathbf{0}$ & $\mathbf{0}$ & $\mathbf{0}$ & $\mathbf{0}$ & 0 \\
\hline & Std. & $\mathbf{0}$ & 0.01249 & $\mathbf{0}$ & $\mathbf{0}$ & $\mathbf{0}$ & 0.01232 \\
\hline \multirow[t]{3}{*}{ F7 } & Ort. & $1.24 \mathrm{E}-09$ & 4.17400 & 2.30824 & $1.38 \mathrm{E}-08$ & $1.50 \mathrm{E}-32$ & 50.28789 \\
\hline & En iyi & $2.73 \mathrm{E}-12$ & 0.63338 & 2.10798 & $1.49 \mathrm{E}-12$ & $1.50 \mathrm{E}-32$ & 17.30331 \\
\hline & Std. & 1.74E-09 & 2.01820 & 0.08005 & $4.92 \mathrm{E}-08$ & $\mathbf{0}$ & 19.57959 \\
\hline \multirow[t]{3}{*}{ F8 } & Ort. & 0.00034 & $1.87 \mathrm{E}-11$ & $1.43 \mathrm{E}-13$ & 1.17E-125 & $3.78 \mathrm{E}-08$ & 26.87974 \\
\hline & En iyi & $2.76 \mathrm{E}-06$ & $1.37 \mathrm{E}-12$ & $5.70 \mathrm{E}-16$ & 3.51E-133 & $1.40 \mathrm{E}-26$ & 17.69641 \\
\hline & Std. & 0.00077 & $2.37 \mathrm{E}-11$ & $1.36 \mathrm{E}-13$ & 4.91E-125 & $2.04 \mathrm{E}-07$ & 5.55862 \\
\hline \multirow[t]{3}{*}{ F9 } & Ort. & 0.00743 & $8.72 \mathrm{E}-10$ & 81.66148 & 0.03955 & $8.53 \mathrm{E}-11$ & 78.42991 \\
\hline & En iyi & $2.18 \mathrm{E}-05$ & $1.95 \mathrm{E}-10$ & 74.91313 & $5.16 \mathrm{E}-05$ & $\mathbf{0}$ & 60.14089 \\
\hline & Std. & 0.02756 & $5.53 \mathrm{E}-10$ & 4.06835 & 0.11534 & 1.69E-10 & 10.14987 \\
\hline \multirow[t]{3}{*}{ F10 } & Ort. & $2.05 \mathrm{E}-08$ & 33.13881 & 137.04358 & 24.19155 & $\mathbf{0}$ & 155.73646 \\
\hline & En iyi & $1.01 \mathrm{E}-11$ & 8.07758 & 108.92262 & 5.38433 & $\mathbf{0}$ & 63.76087 \\
\hline & Std. & $4.73 \mathrm{E}-08$ & 17.99387 & 8.33325 & 10.50401 & $\mathbf{0}$ & 33.66602 \\
\hline
\end{tabular}


Tablo 4. 50 boyutlu test fonksiyonlarında elde edilen sonuçlar

\begin{tabular}{|c|c|c|c|c|c|c|c|}
\hline & & YDAO & EBO & GPİO & GTO & ÖPDO & TSO \\
\hline \multirow{3}{*}{$\mathrm{F} 1$} & Ort. & $1.21 \mathrm{E}-50$ & 0.00193 & $2.27 \mathrm{E}-21$ & $5.70 \mathrm{E}-241$ & 3.52E-267 & $2.18 \mathrm{E}-36$ \\
\hline & En iyi & $5.55 \mathrm{E}-66$ & $9.26 \mathrm{E}-07$ & $5.10 \mathrm{E}-26$ & $5.79 \mathrm{E}-257$ & $4.96 \mathrm{E}-270$ & $3.02 \mathrm{E}-39$ \\
\hline & Std. & $6.54 \mathrm{E}-50$ & 0.00915 & $5.22 \mathrm{E}-21$ & 0 & 0 & $4.23 \mathrm{E}-36$ \\
\hline \multirow{3}{*}{$\mathrm{F} 2$} & Ort. & 0.04310 & 123.45188 & 47.58939 & 42.19588 & 3.77961 & 48.39646 \\
\hline & En iyi & 0.00076 & 27.19353 & 47.03578 & 39.09889 & 0.00040 & 46.14923 \\
\hline & Std. & 0.08878 & 63.91115 & 0.40007 & 2.06084 & 13.57655 & 0.60663 \\
\hline \multirow{3}{*}{ F3 } & Ort. & $4.55 \mathrm{E}-07$ & 0.00011 & 9.16572 & $3.48 \mathrm{E}-06$ & 0 & 6.39201 \\
\hline & En iyi & $8.69 \mathrm{E}-10$ & $4.03 \mathrm{E}-07$ & 8.55662 & $3.04 \mathrm{E}-07$ & $\mathbf{0}$ & 4.64615 \\
\hline & Std. & $9.46 \mathrm{E}-07$ & 0.00026 & 0.20253 & $5.06 \mathrm{E}-06$ & 0 & 0.88287 \\
\hline \multirow{3}{*}{$\mathrm{F} 4$} & Ort. & 0.08959 & 107.62119 & 0 & 0 & $\mathbf{0}$ & 383.93784 \\
\hline & En iyi & $1.46 \mathrm{E}-07$ & 69.64706 & $\mathbf{0}$ & $\mathbf{0}$ & $\mathbf{0}$ & 283.56746 \\
\hline & Std. & 0.28393 & 19.71767 & 0 & $\mathbf{0}$ & 0 & 52.43582 \\
\hline \multirow{3}{*}{ F5 } & Ort. & $3.97 \mathrm{E}-15$ & 2.67599 & $6.41 \mathrm{E}-12$ & 8.88E-16 & $6.50 \mathrm{E}-14$ & 1.08088 \\
\hline & En iyi & $8.88 \mathrm{E}-16$ & 0.01144 & $3.95 \mathrm{E}-13$ & $8.88 \mathrm{E}-16$ & $5.77 \mathrm{E}-14$ & $2.22 \mathrm{E}-14$ \\
\hline & Std. & $1.21 \mathrm{E}-15$ & 1.04502 & $5.96 \mathrm{E}-12$ & 0 & $4.25 \mathrm{E}-15$ & 1.43268 \\
\hline \multirow{3}{*}{ F6 } & Ort. & 0 & 0.02702 & 0 & $\mathbf{0}$ & 0 & 0.00578 \\
\hline & En iyi & 0 & $3.11 \mathrm{E}-06$ & $\mathbf{0}$ & $\mathbf{0}$ & 0 & 0 \\
\hline & Std. & 0 & 0.06199 & 0 & 0 & 0 & 0.00800 \\
\hline \multirow{3}{*}{ F7 } & Ort. & $3.28 \mathrm{E}-07$ & 8.87983 & 4.11957 & 7.33E-06 & $1.50 \mathrm{E}-32$ & 126.87876 \\
\hline & En iyi & $3.19 \mathrm{E}-09$ & 1.99635 & 3.99599 & $8.51 \mathrm{E}-07$ & $1.50 \mathrm{E}-32$ & 44.98305 \\
\hline & Std. & $6.14 \mathrm{E}-07$ & 4.30005 & 0.07264 & $1.07 \mathrm{E}-05$ & 0 & 34.38624 \\
\hline \multirow{3}{*}{ F8 } & Ort. & 0.00242 & $7.52 \mathrm{E}-05$ & $4.39 \mathrm{E}-13$ & 1.32E-122 & $1.18 \mathrm{E}-06$ & 56.68548 \\
\hline & En iyi & $1.75 \mathrm{E}-05$ & $3.33 \mathrm{E}-06$ & $1.59 \mathrm{E}-14$ & 3.29E-131 & $1.90 \mathrm{E}-23$ & 41.54560 \\
\hline & Std. & 0.00249 & 0.00028 & $4.72 \mathrm{E}-13$ & $4.30 \mathrm{E}-122$ & $5.03 \mathrm{E}-06$ & 9.35117 \\
\hline \multirow{3}{*}{ F9 } & Ort. & 0.32004 & 0.00920 & 156.44662 & 0.62138 & 8.38E-05 & 152.84064 \\
\hline & En iyi & 0.00200 & 0.00058 & 146.46686 & 0.04119 & 0 & 116.88283 \\
\hline & Std. & 0.87080 & 0.02111 & 4.08889 & 0.56817 & 0.00033 & 18.65439 \\
\hline \multirow{3}{*}{ F10 } & Ort. & $1.86 \mathrm{E}-06$ & 250.20085 & 284.58709 & 53.09751 & 0 & 324.08870 \\
\hline & En iyi & $1.83 \mathrm{E}-08$ & 51.63057 & 270.91279 & 26.47955 & 0 & 256.90893 \\
\hline & Std. & $6.05 \mathrm{E}-06$ & 376.04332 & 6.07368 & 19.73190 & $\mathbf{0}$ & 56.81305 \\
\hline
\end{tabular}

Şekil 1, Şekil 2 ve Şekil 3' te sırasıyla 10, 30 ve 50 boyutlu F2, F5, F6, F7, F8 ve F9 fonksiyonlarında elde edilen yakınsama grafikleri görülmektedir. Şekil 1 incelendiğinde $\mathrm{F} 2$ için tüm algoritmalar benzer yakınsama göstermektedir. F5' te YDAO, GTO ve ÖPDO yaklaşı 200 iterasyon içerisinde optimum noktaya yakınsadığ fakat geri kalan iterasyonlarda ise yatay seyrettiği görülmektedir. F6' da GPİO, GTO, ÖPDO ve YDAO sırası ile yaklaşık 200, 300, 550 ve 800 . iterasyonlarda optimum noktaya ulaşmaktadır. F7' de ÖPDO çok hızlı bir biçimde yaklaşık 100 iterasyonda optimum noktaya ulaştığ görülmektedir. F8' de GTO her iterasyonda sürekli optimum noktaya yakınsarken geri kalan algoritmalar çok daha yavaş bir yakınsama göstermektedir. F9' da ise sadece ÖPDO' nun optimum noktaya ulaşabildiği görülmektedir. Şekil 2 incelendiğinde F2 için tüm algoritmalar ve F8 için GTO hariç diğer algoritmalar benzer yakınsama göstermektedir. F5' te YDAO, GTO ve ÖPDO yaklaşık 300 iterasyon içerisinde hızlı

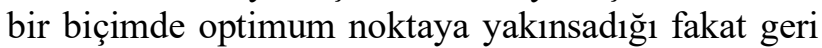
kalan iterasyonlarda ise yatay seyrettiği görülmektedir. F6' da GTO, YDAO ve ÖPDO ise Şekil 1' deki 10 boyutlu fonksiyona göre 30 boyutlu fonksiyonda daha hızlı bir şekilde yaklaşı 150 iterasyon içinde optimum noktaya yakınsadığ görülmektedir. F7' de yine ÖPDO çok hılı bir biçimde yaklaşık 130 iterasyonda optimum noktaya yakınsadığı görülmektedir. F9' da EBO her iterasyonda optimum noktaya doğru yakınsarken diğer algoritmalar genelde yatay seyretmiştir. Fakat ÖPDO yaklaşık ilk 850 iterasyonda yavaş bir biçimde optimum noktaya yakınsarken geri kalan iterasyonlarda çok hızlı bir biçimde optimum noktaya yakınsadığ görülmektedir. Şekil 3 incelendiğinde tüm algoritmalar genel olarak Şekil 2' deki 30 
boyutlu test fonksiyonlarına benzer bir yakınsama göstermişlerdir. Fakat ÖPDO 30 boyutlu F9 fonksiyonunda ilk 850 iterasyonda çok yavaş bir biçimde optimum noktaya yakınsarken geri kalan iterasyonlarda çok hızlı bir biçimde optimum noktaya yakınsamaktaydı. Fakat 50 boyutlu F9 fonksiyonunda her iterasyon yavaş fakat sürekli olarak optimum noktaya yakınsadığ 1 görülmektedir. Tablo 5 ' te ise algoritmaların 50 boyutlu test fonksiyonlarında 1000 iterasyondaki 30 bağımsız çalıștırmada elde edilen ortalama çalıșma süreleri saniye cinsinden verilmiştir. Sonuçlar incelendiğinde YDAO ve TSO' nun süre bakımından en hızlı çalışan algoritmalar olduğu görülmektedir. EBO ve ÖPDO ise en yavaş çalışan algoritmalar olmuştur. Özellikle F7 ve F9 fonksiyonlarında ÖPDO' nun çalışma süresi çok daha uzun sürmektedir.
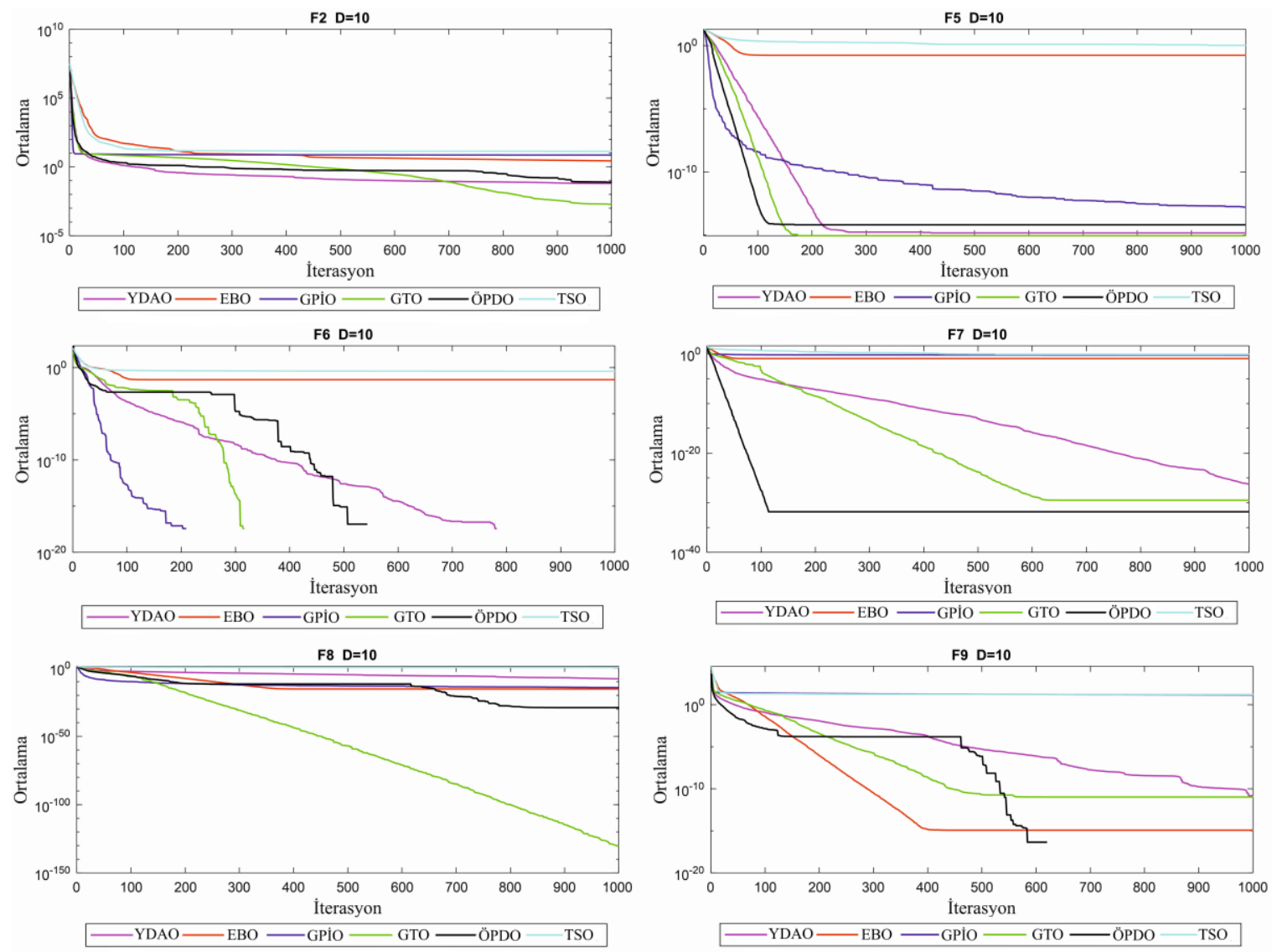

Şekil 1. 10 boyutlu test fonksiyonlarında elde edilen yakınsama grafikleri

Tablo 5. 50 boyutlu test fonksiyonlarında 1000 iterasyonda elde edilen ortalama çalışma süreleri (sn.)

\begin{tabular}{|l|l|l|l|l|l|l|}
\hline Fonksiyon & YDAO & EBO & GPIO & GTO & ÖPDO & TSO \\
\hline F1 & 0.6006 & 10.4534 & 3.6321 & 2.2497 & 5.2366 & 0.7263 \\
\hline F2 & 0.6591 & 9.8305 & 3.6458 & 1.9316 & 8.6151 & 0.8024 \\
\hline F3 & 0.5626 & 9.9163 & 3.3574 & 1.8068 & 7.3788 & 0.7242 \\
\hline F4 & 0.6317 & 10.8736 & 3.7927 & 1.7773 & 7.2985 & 0.7958 \\
\hline F5 & 0.5997 & 10.4969 & 3.3960 & 1.8451 & 6.6720 & 0.7580 \\
\hline F6 & 0.7381 & 10.4719 & 3.4796 & 1.8589 & 10.8462 & 0.8353 \\
\hline F7 & 1.9460 & 11.0680 & 4.6248 & 3.0221 & 52.6764 & 1.8325 \\
\hline F8 & 0.6420 & 9.9106 & 3.3586 & 1.8649 & 6.0859 & 0.8460 \\
\hline F9 & 1.9099 & 11.3575 & 4.9544 & 3.1950 & 64.0522 & 1.9996 \\
\hline F10 & 0.6496 & 10.1604 & 3.5544 & 1.9391 & 7.9229 & 0.8512 \\
\hline
\end{tabular}




\section{DOI: $10.29132 /$ jpas.855869}
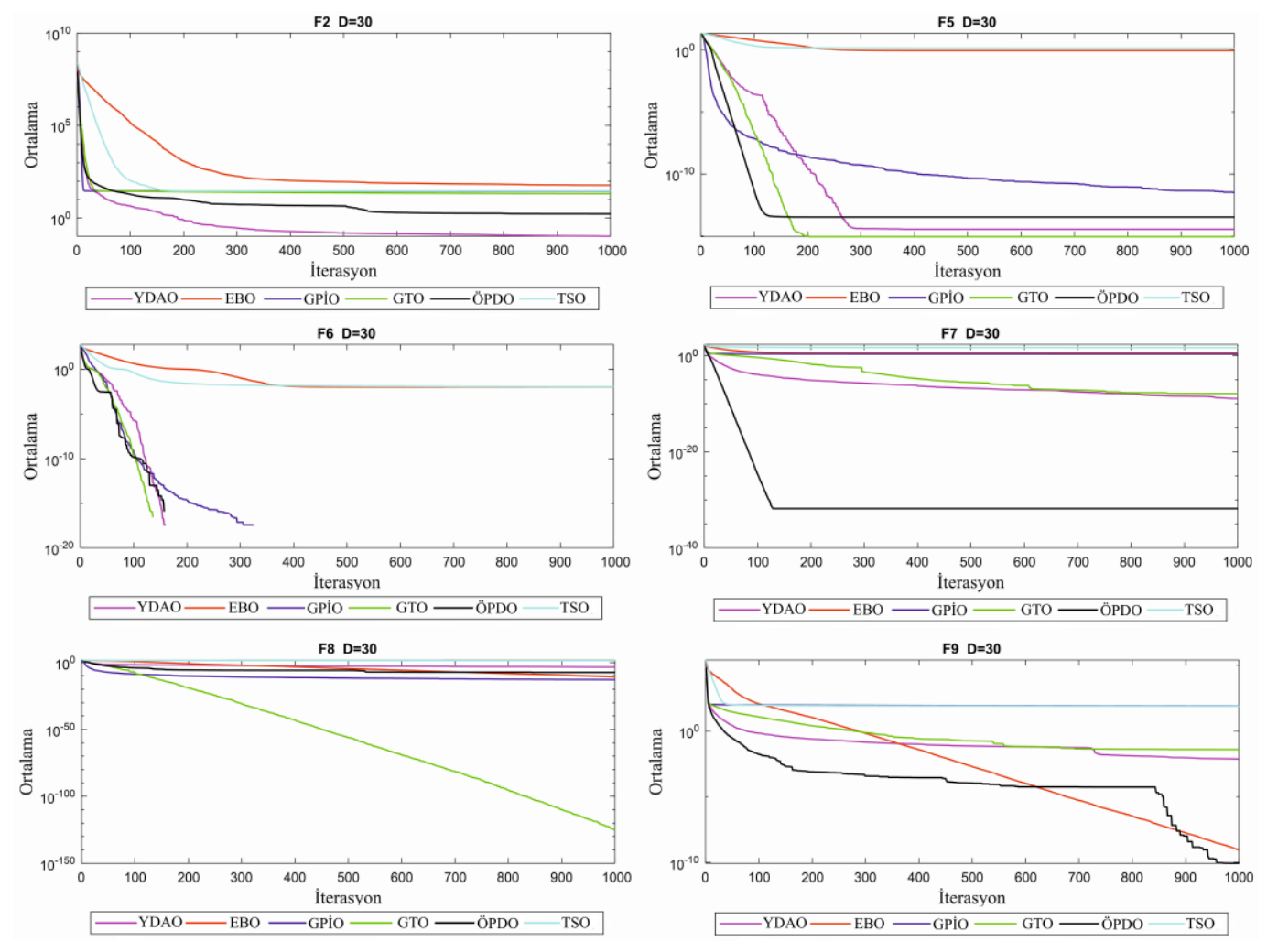

Şekil 2. 30 boyutlu test fonksiyonlarında elde edilen yakınsama grafikleri

\section{SONUÇ}

Metasezgisel algoritmalar birçok optimizasyon problemi için kabul edilebilir sürelerde optimuma yakın çözümler veren, klasik algoritmalar gibi ilgilenilen problemler üzerinde değişiklik yapmadan o problemlere kolaylıkla uyarlanabilen, farklı tipteki karar değişkenleri ve sınırlayıcıların olması durumunda bile çözüm stratejileri sunabilen algoritmalardır. Bu avantajlarından dolayı son birkaç on yıldır araştırmacılar birçok metasezgisel algoritma önermiş̧ir ve halen yeni algoritmalarda literatüre kazandırılmaya devam etmektedir. Bu çalışmada en yeni metasezgisel algoritmalardan olan YDAO, EBO, GPİ, GTO, ÖPDO ve TSO' nun performansları 10, 30 ve 50 boyutlu 10 farklı matematiksel kalite test fonksiyonu kullanılarak ayrıntılı bir biçimde test edilmiştir. Test sonuçlarına göre, bu 10 matematiksel fonksiyondan 7 tanesinde en iyi çözümleri veren
ÖPDO' nun en başarılı algoritma olduğu görülmüştür. GTO ise 4 fonksiyonda en iyi sonuçları verdiği görülmüştür. YDAO ve GPIOO ise 2'şer fonksiyon en iyi çözümleri vermiştir. En kötü performansı ise EBO ve TSO göstermiştir. ÖPDO her ne kadar en iyi çözümleri üretmişse de çalışma süresi diğer algoritmalara göre daha uzun sürmektedir. $\mathrm{Bu}$ durum büyük optimizasyon problemlerinin çözümünde bir dezavantaj olabilir.

$\mathrm{Bu}$ çalışma, araştırmacıların en güncel metasezgisel optimizasyon algoritmalarından bazılarına kolaylıkla bir arada ulaşması açısından hem de araştırmacılara kendi çalışmalarında kullanabilecekleri metasezgisel algoritmaların performansları hakkında bir ön fikir vererek uygun algoritmayı seçmesine yardımcı olması açısından önemlidir. 

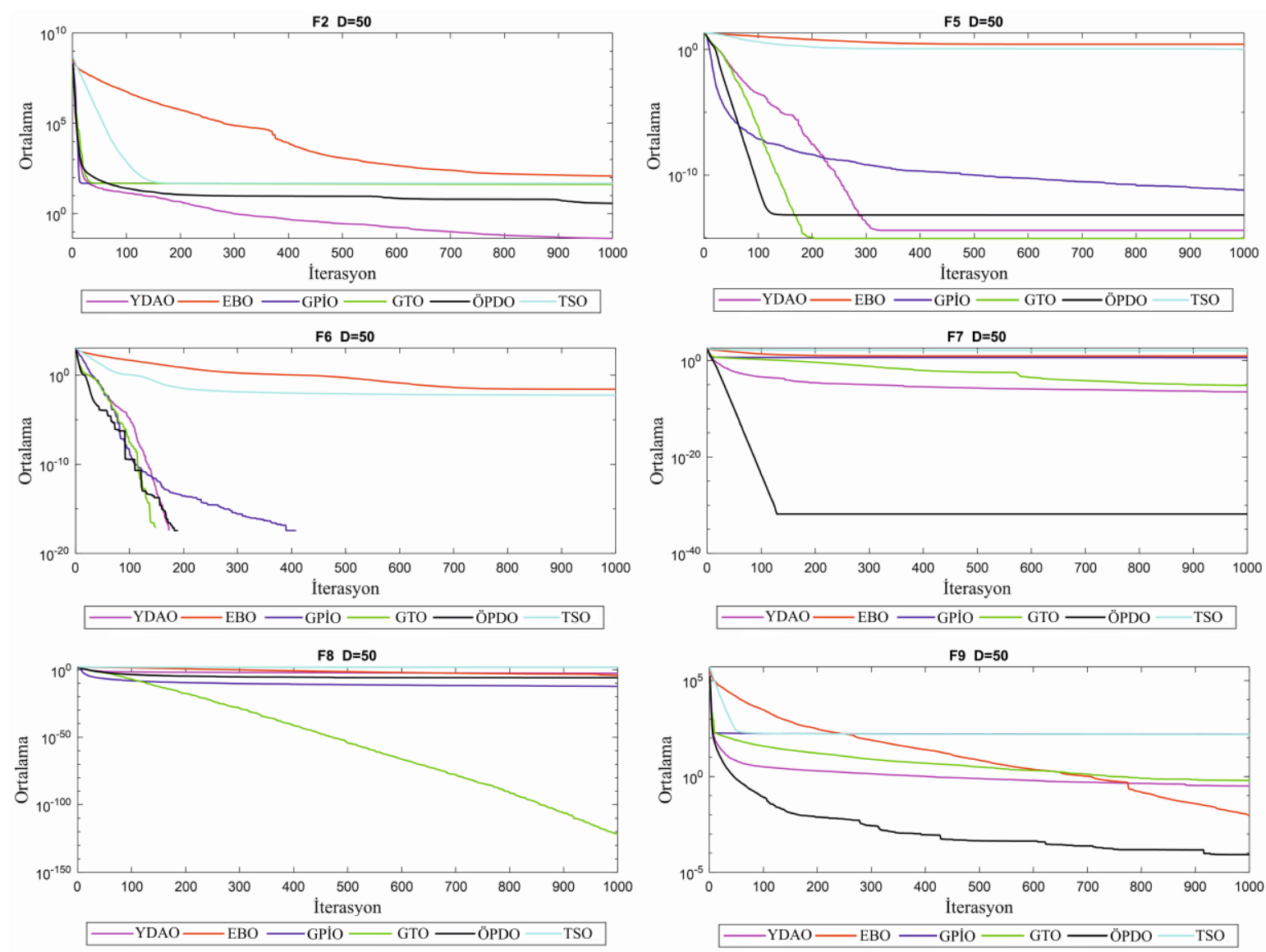

Şekil 3. 50 boyutlu test fonksiyonlarında elde edilen yakınsama grafikleri

\section{ÇIKAR ÇATIŞMASI BEYANI}

Yazarlar bu makale ile ilgili herhangi bir çıkar çatışması bildirmemektedir.

\section{ARAŞTIRMA VE YAYIN ETİĞİ BEYANI}

Yazarlar bu çalışmanın araştırma ve yayın etiğine uygun olduğunu beyan eder.

\section{KAYNAKLAR}

Ahmadianfar, I., Bozorg-Haddad, O. ve Chu, X. (2020). Gradient-based optimizer: A new Metaheuristic optimization algorithm. Information Sciences, 540, 131-159.

Alatas, B. (2012). A novel chemistry based metaheuristic optimization method for mining of classification rules. Expert Systems with Applications, 39(12), 11080-11088.

Alatas, B., Akin, E. ve Ozer, A. B. (2009). Chaos embedded particle swarm optimization algorithms. Chaos, Solitons \& Fractals, 40(4), 1715-1734.

Altunbey, F. ve Alataş, B. (2015). Sosyal ağ analizi için sosyal tabanlı yapay zekâ optimizasyon algoritmalarının incelenmesi. International Journal of Pure and Applied Sciences, 1(1), 33-52.

Ashrafi, S. M. ve Dariane, A. B. (2011). A novel and effective algorithm for numerical optimization: melody search (MS). In 2011 11th international conference on hybrid intelligent systems (HIS) (pp. 109-114). IEEE.

Birbil, Ş. İ. ve Fang, S. C. (2003). An electromagnetismlike mechanism for global optimization. Journal of Global Optimization, 25(3), 263-282.

Borji, A. ve Hamidi, M. (2009). A new approach to global optimization motivated by parliamentary political competitions. International Journal of Innovative Computing, Information and Control, 5(6), 16431653.

Can, Ü. ve Alataş, B. (2015). Bitki zekâsında yeni bir alan: kök kütlesi optimizasyonu. Türk Doğa ve Fen Dergisi, 8.

Chou, J. S. ve Truong, D. N. (2021). A novel metaheuristic optimizer inspired by behavior of jellyfish in ocean. Applied Mathematics and Computation, 389, 125535.

Das, B., Mukherjee, V. ve Das, D. (2020). Student psychology based optimization algorithm: A new population based optimization algorithm for solving 
optimization problems. Advances in Engineering Software, 146, 102804.

Gao, S. ve De Silva, C. W. (2018). Estimation distribution algorithms on constrained optimization problems. Applied Mathematics and Computation, 339, 323345.

Harifi, S., Mohammadzadeh, J., Khalilian, M. ve Ebrahimnejad, S. (2020). Giza Pyramids Construction: an ancient-inspired metaheuristic algorithm for optimization. Evolutionary Intelligence, 1-19.

Holland, J. H. (1992). Adaptation in natural and artificial systems: an introductory analysis with applications to biology, control, and artificial intelligence. MIT press.

Jamil, M. ve Yang, X. S. (2013). A literature survey of benchmark functions for global optimisation problems. International Journal of Mathematical Modelling and Numerical Optimisation, 4(2), 150194.

Karaboga, D. ve Akay, B. (2009). A comparative study of artificial bee colony algorithm. Applied Mathematics And Computation, 214(1), 108-132.

Kashan, A. H. (2014). League Championship Algorithm (LCA): An algorithm for global optimization inspired by sport championships. Applied Soft Computing, 16, 171-200.

Kaur, S., Awasthi, L. K., Sangal, A. L. ve Dhiman, G. (2020). Tunicate swarm algorithm: a new bioinspired based metaheuristic paradigm for global optimization. Engineering Applications of Artificial Intelligence, 90, 103541.

Kennedy, J. ve Eberhart, R. (1995). Particle swarm optimization. In Proceedings of ICNN'95international conference on neural networks (Vol. 4, pp. 1942-1948). IEEE.

Kizıloluk, S. ve Özer, A. B. (2016). Melez elektromanyetizma benzeri-parçacık sürü optimizasyon algoritması. Dicle Üniversitesi Mühendislik Fakültesi Mühendislik Dergisi, 7(3), 515-526.

Lee, K. S. ve Geem, Z. W. (2005). A new meta-heuristic algorithm for continuous engineering optimization: harmony search theory and practice. Computer Methods in Applied Mechanics and Engineering, 194(36-38), 3902-3933.

Mirjalili, S. ve Lewis, A. (2016). The whale optimization algorithm. Advances in Engineering Software, 95, 51-67.

Ong, K. M., Ong, P. ve Sia, C. K. (2021). A carnivorous plant algorithm for solving global optimization problems. Applied Soft Computing, 98, 106833.

Osaba, E., Diaz, F. ve Onieva, E. (2014). Golden ball: a novel meta-heuristic to solve combinatorial optimization problems based on soccer concepts.Applied Intelligence, 41(1), 145-166.
Qi, X., Zhu, Y., Chen, H., Zhang, D. ve Niu, B. (2013). An idea based on plant root growth for numerical optimization. In International Conference on Intelligent Computing (pp. 571-578). Springer, Berlin, Heidelberg.

Rao, R. V., Savsani, V. J. ve Vakharia, D. P. (2012). Teaching-learning-based optimization: an optimization method for continuous non-linear large scale problems. Information Sciences, 183(1), 1-15.

Rashedi, E., Nezamabadi-Pour, H. ve Saryazdi, S. (2009). GSA: a gravitational search algorithm. Information Sciences, 179(13), 2232-2248.

Sacco, W. F. ve Oliveira, C. R. D. (2005). A New Stochastic Optimization Algorithm based on a Particle Collision Metaheuristic. 6th World Congresses of Structural and Multidisciplinary Optimization, Rio de Janerio, Brazil.

Storn, R. ve Price, K. (1997). Differential evolution-a simple and efficient heuristic for global optimization over continuous spaces. Journal of Global Optimization, 11(4), 341-359.

Xie, L., Zeng, J. ve Cui, Z. (2009). General framework of artificial physics optimization algorithm. In 2009 World Congress on Nature \& Biologically Inspired Computing (NaBIC) (pp. 1321-1326). IEEE.

Yang, X. S. ve Gandomi, A. H. (2012). Bat algorithm: a novel approach for global engineering optimization. Engineering Computations, 29(5), 464-483.

Yang, X. S. (2012). Flower pollination algorithm for global optimization. In International conference on unconventional computing and natural computation (pp. 240-249). Springer, Berlin, Heid 